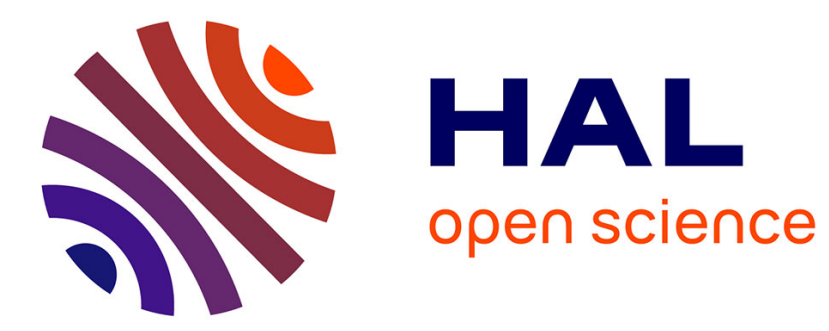

\title{
Étude de transferts d'excitation dans un plasma induit par haute fréquence entre gaz plasmagène et éléments introduits
}

\author{
J.M. Mermet, C. Trassy
}

\section{To cite this version:}

J.M. Mermet, C. Trassy. Étude de transferts d'excitation dans un plasma induit par haute fréquence entre gaz plasmagène et éléments introduits. Revue de Physique Appliquée, 1977, 12 (9), pp.12191222. 10.1051/rphysap:019770012090121900 . jpa-00244302

HAL Id: jpa-00244302

https://hal.science/jpa-00244302

Submitted on 1 Jan 1977

HAL is a multi-disciplinary open access archive for the deposit and dissemination of scientific research documents, whether they are published or not. The documents may come from teaching and research institutions in France or abroad, or from public or private research centers.
L'archive ouverte pluridisciplinaire $\mathbf{H A L}$, est destinée au dépôt et à la diffusion de documents scientifiques de niveau recherche, publiés ou non, émanant des établissements d'enseignement et de recherche français ou étrangers, des laboratoires publics ou privés. 


\title{
Classification \\ Physics Abstracts \\ 52.50 \\ ÉTUDE DE TRANSFERTS D'EXCITATION DANS UN PLASMA INDUIT PAR HAUTE FRÉQUENCE ENTRE GAZ PLASMAGÈNE ET ÉLÉMENTS INTRODUITS (*)
}

\author{
J. M. MERMET et C. TRASSY
}

Laboratoire de chimie industrielle et analytique bt 401, Institut National des Sciences Appliquées

20, avenue A.-Einstein, 69621 Villeurbanne Cedex, France

(Reçu le 30 décembre 1976, accepté le 25 mai 1977)

\begin{abstract}
Résumé. - L'étude de l'excitation des éléments injectés dans un plasma d'argon induit par haute fréquence à la pression atmosphérique montre que l'hypothèse de l'E. T. L. n'est pas vérifiée. Ce fait est confirmé par la différence obtenue entre la température d'ionisation et celles d'excitation. L'excitation de certaines raies des éléments injectés peut s'expliquer par l'action des métastables de l'argon neutre et de l'argon ionisé. C'est pourquoi une méthode d'absorption atomique à deux longueurs d'onde a été mise au point qui permet d'effectuer la mesure des densités de métastables.
\end{abstract}

\begin{abstract}
Studies of injected elements in an inductively coupled argon plasma working at atmospheric pressure show a departure from $\mathrm{L}$. T. E. This is confirmed by the discrepancies between the ionization temperature and the excitation temperatures. The excitation of some lines of these elements can be explained through the action of metastable levels of neutral and ionized argon. An atomic absorption experiment using a two-line method has been set up in order to measure the metastable densities.
\end{abstract}

1. Introduction. - Le plasma produit par couplage inductif à la pression atmosphérique est essentiellement utilisé comme source lumineuse en analyse par spectroscopie d'émission [1-3]. Les limites de détection obtenues (de l'ordre du $\mu \mathrm{g} .1^{-1}$ ) et les linéarités des courbes de dosage $\left(10^{3}\right.$ à $\left.10^{4}\right)$ sont meilleures que celles obtenues avec les autres méthodes optiques classiques [4]. De plus, les différents effets inter-éléments y sont très faibles.

Nous avons utilisé successivement deux générateurs fonctionnant l'un à $5 \mathrm{MHz}$, l'autre à $40 \mathrm{MHz}$ avec une puissance appliquée variant de 0,5 à $4 \mathrm{~kW}$ [3]. L'oscillateur est du type à circuit oscillant à self et capacité pour le premier, à lignes accordées pour le second. L'inducteur est composé de cinq spires en cuivre refroidies par eau. La torche (Fig. 1) permet d'obtenir un débit minimum d'argon tout en conduisant à de bonnes performances analytiques. L'injection d'éléments se fait à partir de solutions liquides nébulisées par ultrasons [5]. Ce dispositif est associé à un système de désolvatation [6] afin de supprimer la présence d'eau dans la décharge. Les conditions opératoires sont résumées dans le tableau de la figure 1.

(*) Communication présentée au Congrès National de Physique des Plasmas, Paris, 6-10 décembre 1976.

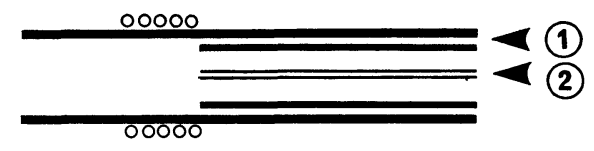

Fig. 1. - Schéma de principe de la torche à plasma : 1) injection d'argon plasmagène ; 2) injection d'éléments. Caractéristiques de fonctionnement du plasma

\begin{tabular}{|ll|}
\hline Fréquence & $40 \mathrm{MHz}$ \\
Puissance Appliquée & $1,8 \mathrm{~kW}$ \\
Hauteur de l'inducteur & $50 \mathrm{~mm}$ \\
Diamètre extérieur de la torche & $30 \mathrm{~mm}$ \\
Débit de gaz plasmagène & $151 . \mathrm{min} .^{-1}$ \\
\hline
\end{tabular}

[Block diagram of the torch configuration ; 1) Plasma gas injection ; 2) aerosol carrier gas injection.]

2. Etude des températures. - Alors qu'il est reconnu que les plasmas à basse pression sont hors ETL, le plasma HF à pression atmosphérique a longtemps été considéré en ETL ou très proche de l'ETL à l'instar des plasmas-arcs. Des mesures de température et de densité électronique ont été faites aussi bien sur les éléments injectés ( $\mathrm{Fe}$, Ti par exemple) que sur l'argon plasmagène. Les températures d'excitation ont été obtenues en prenant la pente de la droite $\log I \lambda / g A$ en fonction de l'énergie d'excitation après sélection des probabilités de transition [7] (méthode du diagramme 
atomique de Boltzmann). Elles sont en bon accord entre elles.

Les densités électroniques ont été déterminées par l'élargissement des raies d'argon par effet Stark lorsque celui-ci prédomine $[7,8]$. La température d'ionisation déduite de la densité électronique à l'aide de l'équation de Saha diffère notablement de la température d'excitation des éléments et de l'argon (Tableau I). Cela indique l'absence d'E. T. L. dans cette source.

\section{TABLEAU I}

Comparaison des températures d'excitation et d'ionisation pour deux générateurs de plasma haute fréquence.

$\begin{array}{ccccc}\text { Générateur } & \text { Elément } & T_{\text {exc }} & n_{\mathrm{e}}(\text { Stark }) & T_{\text {ion }} \\ \overline{-} & \overline{\mathrm{Ar}} & 5000 \mathrm{~K} & 5 \times \overline{10^{14} \mathrm{~cm}^{-3}} & 7350 \mathrm{~K} \\ 40 \mathrm{~Hz} & \mathrm{Fe} & 4800 & & \\ & \mathrm{Ti} & 4800 & & \\ 5 \mathrm{MHz} & \mathrm{Ar} & 6200 \mathrm{~K} & 3,5 \times 10^{15} \mathrm{~cm}^{-3} & 8600 \mathrm{~K} \\ & \mathrm{Fe} & 6000 & & \\ & \mathrm{Ti} & 6200 & & \end{array}$

3. Etude de l'excitation. - Pour obtenir les meilleures limites de détection, les différents auteurs $[1,9]$ utilisent, ainsi que nous-même $[3,6]$, des raies d'éléments une fois ionisé bien que la température d'excitation dans ces plasmas soit inférieure à $6000 \mathrm{~K}$. Si l'équilibre thermodynamique était respecté, les raies I devraient être les plus intenses. Dans la pratique, les meilleurs résultats analytiques sont obtenus pour des raies dont l'énergie d'ionisation ou la somme des énergies d'ionisation et d'excitation est inférieure à environ $11 \mathrm{eV}$. Dans le cas des halogènes dont les énergies d'ionisation sont élevées $(\mathrm{Br} 11,84 \mathrm{eV}, \mathrm{Cl} 13,01 \mathrm{eV}$, F $17,42 \mathrm{eV}$ ) l'excitation est alors très faible. C'est pourquoi l'effet Penning et le transfert d'excitation ont été proposés [10-12] comme mécanismes d'excitation importants : ils mettent en œuvre les niveaux métastables de l'argon neutre, selon des réactions telles que :

$$
\begin{aligned}
\mathrm{Ar}^{\text {méta }}+\mathrm{X} \rightarrow & \mathrm{Ar}+\mathrm{X}^{+*}+\mathrm{e} \\
& \mathrm{Ar}+\mathrm{X}^{+}+\mathrm{e}
\end{aligned}
$$

Par ailleurs certaines raies d'éléments introduits dont l'énergie d'excitation est plus élevée que l'énergie d'ionisation de l'argon ont été observées : Al II $358,6 \mathrm{~nm}(21,29 \mathrm{eV}), \mathrm{Br}$ II $470,4 \mathrm{~nm}(26,04 \mathrm{eV})$, S II 471,6 nm (26,60 eV), Cl II 479,4 nm (28,97 eV), Ti III 251,6 nm (29,44 eV). Dans le cas du soufre, il est à remarquer qu'une cinquantaine de nouvelles raies ont ainsi été excitées [15]. Une telle excitation $\left(^{1}\right)$ peut s'interpréter selon un transfert d'énergie à partir des niveaux métastables de l'argon ionisé ${ }^{4} \mathrm{D}_{7 / 2},{ }^{4} \mathrm{~F}_{9 / 2}$, ${ }^{4} F_{7 / 2},{ }^{2} F_{7 / 2}$ dont l'énergie est comprise entre 32 et $34 \mathrm{eV}$ au-dessus du fondamental.

$$
A r^{+ \text {uéta }}+X \rightarrow\left\{\begin{array}{l}
A r+X^{+*} \\
A r+X^{2+*}+e .
\end{array}\right.
$$

(1) Strictement inexplicable dans l'hypothèse de l'ETL.
Parallèlement à l'étude directe du plasma, nous avons fait une étude qualitative de l'influence du gaz utilisé à l'aide de lampes à cathode creuse spécialement construites au laboratoire pour cet usage. L'intensité des spectres des raies II et III par rapport au spectre de raies I varie en fonction de la nature du gaz de transfert. En effet bien que l'énergie maximum obtenue dans une lampe à cathode creuse soit plus faible pour l'argon $(\simeq 70 \mathrm{eV})$ que pour le néon $(\simeq 110 \mathrm{eV})$, les raies II et III des éléments sont plus intenses avec l'argon qu'avec le néon. Cela peut s'expliquer par le fait que, contrairement à l'argon ionisé, le néon ionisé ne possède pas de niveaux métastables.

4. Méthode de mesure utilisée. - Nous avons cherché à déterminer directement la population des niveaux métastables. Pour cela, nous utilisons l'absorption atomique qui permet d'accéder aux populations des niveaux inférieurs des transitions. Cette méthode a déjà été employée $[15,16]$. La source primaire utilisée est généralement un plasma d'argon ou une lampe à xénon. Dans notre cas, nous avons recours à une lampe à cathode creuse à l'argon. Cette source présente l'avantage de fournir des raies dont la largeur est beaucoup plus faible que celle des raies du plasma HF. En contre partie, la luminosité faible vis-à-vis de celle du plasma exige un dispositif de détection performant. L'intensité du plasma est en effet pour une même longueur d'onde 30 à 200 fois plus forte, suivant la puissance, que celle de la lampe à cathode creuse (cathode en aluminium).

Nous utilisons une méthode de mesure à deux longueurs d'onde $[17,3]$ dont le principe est représenté sur la figure 2. Les deux radiations sont issues de la

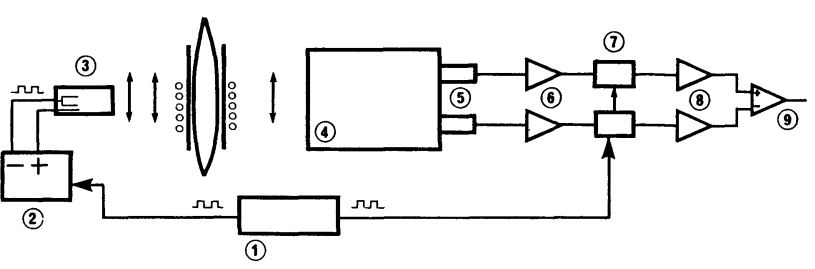

FIG. 2. - Schéma de principe de la méthode d'absorption atomique à deux longueurs d'ondes: 1) oscillateur pilote, 2) alimentation lampe, 3) lampe à cathode creuse, 4) polychromateur, 5) photomultiplicateurs, 6) amplificateurs, 7) détection synchrone, 8) étages logarithmiques, 9) mesure.

[Block diagram of the two line atomic absorption method: 1) Reference oscillator, 2) lamp power supply, 3) hollow cathode lamp, 4) polychromator, 5) photomultipliers, 6) amplifiers, 7) lock in amplifiers, 8) logarithmic amplifiers, 9) read out system.]

même source : l'une correspond à une transition vers un niveau métastable et sert à la mesure de l'absorption résonante, l'autre radiation non susceptible d'absorption résonante sert de référence. Les deux radiations subissent les perturbations non sélectives dues au plasma : absorption de fond, diffraction... Le signal de la lampe à cathode creuse est modulé électronique- 
ment par l'intermédiaire du courant de décharge. Ce procédé a l'avantage sur les modulations optiques classiques d'offrir une excellente stabilité de la phase du signal, ce qui est capital pour l'efficacité des dispositifs à détection synchrone. Le même oscillateur pilote la modulation du faisceau et la détection. La comparaison des deux signaux permet de déterminer la valeur de l'absorption résonante. La mesure des intensités des deux radiations est simultanée, le dispositif dispersif ayant la possibilité de fonctionner en polychromateur.

Les difficultés opératoires supplémentaires qu'introduit le plasma HF par comparaison avec les plasmasarcs sont de deux types : le champ électromagnétique HF est très intense, les perturbations qu'il introduit (courants induits se superposant aux courants de signaux) peuvent être atténuées mais non supprimées par un blindage rigoureux de toute l'installation de mesure. D'autre part, l'accès aux populations dans la zone où s'effectue l'excitation des éléments impose d'observer le plasma à travers les spires de l'inducteur et à travers le tube de quartz constituant l'enceinte. La précision des mesures s'en trouve altérée.

5. Choix des raies. - La population d'un niveau $N_{\mathrm{m}}$ d'une transition $\mathrm{n} \rightarrow \mathrm{m}$ peut être calculée à partir de la mesure du coefficient d'absorption intégral par la relation :

$$
\begin{aligned}
K & =\int_{0}^{\infty} k(\lambda) \mathrm{d} \lambda \\
& =\frac{\lambda^{4}}{8 \Pi c} \frac{g_{\mathrm{n}}}{g_{\mathrm{m}}} A_{\mathrm{nm}} N_{\mathrm{m}}\left(1-\frac{g_{\mathrm{m}} N_{\mathrm{m}}}{g_{\mathrm{n}} N_{\mathrm{m}}}\right)
\end{aligned}
$$

c vitesse de la lumière, $g$ poids statistique du niveau considéré, $N$ population du niveau considéré.

Par ailleurs, le coefficient central linéaire d'absorption $k_{0}$ peut être relié à $K$ par la relation

$$
K=a \cdot k_{0} \cdot \Delta \lambda_{\mathrm{R}}
$$

où $\Delta \lambda_{R}$ est la largeur à mi-hauteur de la raie résultant des effets Doppler, Lorentz et Stark et $a$ le coefficient de forme de la raie.

Dans le cas où l'on néglige le terme correctif $\left(1-\left(g_{\mathrm{m}} N_{\mathrm{n}} / g_{\mathrm{n}} N_{\mathrm{m}}\right)\right)$ dû à l'émission induite (ce qui, comme l'indiquent les résultats, est parfaitement justifié), la population du niveau $\mathrm{m}$ est donnée par:

$$
N_{\mathrm{m}}=\frac{8 \Pi c}{\lambda^{4}} \frac{g_{\mathrm{m}}}{g_{\mathrm{n}}} \frac{1}{A_{\mathrm{nm}}} k_{0} \cdot \Delta \lambda_{\mathrm{R}} \cdot a .
$$

Les raies utilisées pour les niveaux métastables de l'argon I sont les raies $415,85 \mathrm{~nm}$ et $696,54 \mathrm{~nm}$. Elles ont été choisies car leur coefficient d'absorption permet de couvrir une échelle importante de densité de population (Tableau II). On n'a utilisé de référence (Al I. $396,1 \mathrm{~nm}$ ) que pour la première raie.

\section{TABLEAU II}

Caractéristiques calculées des raies pour $T_{\mathrm{exc}}=5000 \mathrm{~K}$ et $n_{\mathrm{e}}=5 \times 10^{14} \mathrm{~cm}^{-3}$ (Températures d'excitation et densité électronique mesurées sur l'argon dans les conditions expérimentales) (voir Tableaux 1 et 2).

$\begin{array}{cccl}\frac{\lambda}{415,86} \mathrm{~nm} & \text { Transition } & & \\ & 3 \mathrm{p}^{5} 5 \mathrm{p} \rightarrow 3 \mathrm{p}^{5} 4 \mathrm{~s} & \Delta \lambda_{\mathrm{D}} & 3,33 \times 10^{-3} \mathrm{~nm} \\ & & \Delta \lambda_{\mathrm{L}} & 0,08 \times 10^{-3} \mathrm{~nm} \\ & & \Delta \lambda_{\mathrm{S}} & 0,89 \times 10^{-3} \mathrm{~nm} \\ & & \Delta \lambda_{\mathrm{R}} & 3,66 \times 10^{-3} \mathrm{~nm} \\ & & \text { déplacement } & 0,38 \times 10^{-3} \mathrm{~nm} \\ 696,54 \mathrm{~nm} & 3 \mathrm{p}^{5} 4 \mathrm{p} \rightarrow 3 \mathrm{p}^{5} 4 \mathrm{~s} & k_{0} / N_{\mathrm{m}} & 1,1 \times 10^{-18} \mathrm{~m}^{-1} \\ & & \Delta \lambda_{\mathrm{D}} & 5,6 \times 10^{-3} \mathrm{~nm} \\ & & \Delta \lambda_{\mathrm{L}} & 0,23 \times 10^{-3} \mathrm{~nm} \\ & & \Delta \lambda_{\mathrm{S}} & 0,42 \times 10^{-3} \mathrm{~nm} \\ & & \Delta \lambda_{\mathrm{R}} & 6,47 \times 10^{-3} \mathrm{~nm} \\ & & \text { déplacement } & 0,34 \times 10^{-3} \mathrm{~nm} \\ & & k_{0} / N_{\mathrm{m}} & 1,7 \times 10^{-17} \mathrm{~m}^{-1} \\ & & & \end{array}$

6. Résultats. - Les premières mesures effectuées sur les raies 415,86 et $696,54 \mathrm{~nm}$ ont permis de déterminer une densité de population du niveau $1 \mathrm{~S}^{5} \mathrm{com}$ prise entre $6 \times 10^{11} \mathrm{~cm}^{-3}$ et $3 \times 10^{12} \mathrm{~cm}^{-3}$ au centre de la torche pour une puissance appliquée de $2,1 \mathrm{~kW}$. La population de ce niveau tombe en dessous de $10^{11} \mathrm{~cm}^{-3}$ à une distance de $1,5 \mathrm{~cm}$ au-dessus de l'inducteur.

Si cette valeur est assez proche de celles mesurées dans des plasmas-arcs [16], elle traduit en fait un écart considérable à l'ETL qu'on n'observe pas dans ces derniers. Les températures d'excitation mesurées sur l'argon sont très différentes : $5000 \pm 200 \mathrm{~K}$ pour le plasma $\mathrm{HF} 40 \mathrm{MHz}, 9600$ à $10800 \mathrm{~K}$ suivant le courant de décharge pour le plasma arc [16]. En supposant l'ETL dans le cas du plasma $\mathrm{HF}$, la densité aurait dû être de $6 \times 10^{6} \mathrm{~cm}^{-3}$.

En ce qui concerne les métastables $\mathrm{Ar}^{+}$, les mesures qui ont été tentées sur la raie 401,37 nm (référence Ar II. 392,67 nm) ne permettent à l'heure actuelle ni de confirmer ni d'infirmer les hypothèses avancées, car elles manquent de reproductibilité et sont à la limite des possibilités de la méthode. En tenant compte des probabilités de transition fournies par la littérature et des performances de l'appareillage utilisé, la densité limite détectable $\mathrm{d}^{\prime} \mathrm{Ar}^{+ \text {méta }}$ se situerait aux environs de $10^{9} \mathrm{~cm}^{-3}$.

\section{Bibliographie}

[1] Fassel, V. A., Kniseley, R. N., Anal. Chem. 46 (1974) $1110 \mathrm{~A}$.

[2] Greenfield, S., Mc Geachin, H. McD., Smith, P. B., Talanta 23 (1976) 1.

[3] Abdallah, M. H., Diemtaszonek, R., Jarosz, J., Mermet,
J. M., Robin, J., Trassy, C., Anal. Chim. Acta 84 (1976) 271.

[4] Winefordner, J. D., FitzGerald, J. J., OMEnetto, N., Appl. Spectros. 29 (1975) 369.

[5] Mermet, J. M., Robin, J., Anal. Chem. 40 (1968) 1918. 
[6] Souilliart, J. C., Robin, J., Analusis 1 (1972) 427.

[7] Mermet, J. M., Spectrochim. Acta 30 B (1975) 383.

[8] Jarosz, J., Mermet, J. M., Robin, J., C. R. Hebd. Séan. Acad. Sci. Série B 278 (1974) 885.

[9] Boumans, P. W. J. M., de Boer, J. F., Spectrochim. Acta 30 B (1975) 309.

[10] Bush, K. W., Vickers, T. J., Spectrochim. Acta 28 B (1973) 85.

[11] Braman, R. S., Dynako, A., Anal. Chem. 40 (1968) 95.

[12] Mermet, J. M., C. R. Hebd. Séan. Acad. Sci. Série B 281 (1975) 273.
[13] Jarosz, J., Mermet, J. M., 17 (1977) 237. J. Quant. Spectrosc. Radiat. Transfer.

[14] Delcroix, J. L., Matos-Ferreira, C., Ricard, A., Atomes et Molécules Métastables dans les gaz ionisés, (Editions du CNRS, Paris) 1975.

[15] Ranson, P., Chapelle, J., J. Physique Colloq. 32 (1971) C5b-39.

[16] BaCri, J., Gleizes, A., Gomes, A. M., J. Quant. Spectrosc. Radiat. Transfer. 15 (1975) 7.

[17] Trassy, C., Thèse Dr Ing. Université Claude Bernard, Lyon (1976). 